\title{
SMALL DEGENERATE SIMPLICES CAN BE BAD FOR SIMPLEX METHODS
}

\author{
Shinji Mizuno \\ Tokyo Institute of Technology
}

\author{
Noriyoshi Sukegawa \\ Chuo University
}

\author{
Antoine Deza \\ McMaster University
}

(Received August 2, 2016; Revised December 21, 2016)

\begin{abstract}
We show that the simplex method with Dantzig's pivoting rule may require an exponential number of iterations over two highly degenerate instances. The feasible region of the first instance is a full dimensional simplex, and a single point for the second one. In addition, the entries of the constraint matrix, the right-hand-side vector, and the cost vector are $\{0,1,2\}$-valued. Those instances, with few vertices and small input data length, illustrate the impact of degeneracy on simplex methods.
\end{abstract}

Keywords: Linear programming, simplex methods, small degenerate instances

\section{Introduction}

While simplex methods are highly efficient in practice for solving linear optimization, many instances requiring an exponential number of iterations are known. One such instance is the Klee-Minty cube [6] and its variants. In dimension $m$, the simplex method visits all the $2^{m}$ non-degenerate basic feasible solutions corresponding to the vertices of the Klee-Minty cube. Thus, the simplex method requires $2^{m}-1$ iterations.

In this note, we essentially perturb the right-hand-side of a Klee-Minty cube considered by Kitahara and Mizuno [4, 5] so that the feasible region becomes a full dimensional simplex. Further perturbing the right-hand-side, the feasible region is reduced to a zero-dimensional simplex, i.e. a single point. Let $\left(L O_{0}\right)$ denote the linear optimization instance considered by Kitahara and Mizuno, and $\left(\mathrm{LO}_{1}\right)$ and $\left(\mathrm{LO}_{2}\right)$ the instances obtained by perturbing the right-hand-side of $\left(L O_{0}\right)$. We observe that the analysis of Kitahara and Mizuno, showing that $\left(L O_{0}\right)$ requires $2^{m}-1$ iterations, can be adapted to show that $\left(L O_{1}\right)$ and $\left(L O_{2}\right)$ require, respectively, $2^{m-1}+1$ and $2^{m}-1$ iterations. For both $\left(L O_{1}\right)$ and $\left(L O_{2}\right)$, an exponential number of iterations are performed at a single degenerate vertex. In addition, the entries of the constraint matrix, the right-hand-side vector, and the cost vector are $\{0,1,2\}$-valued for both $\left(L O_{1}\right)$ and $\left(L O_{2}\right)$. Those instances, with few vertices and small input data length, illustrate the impact of degeneracy on simplex methods, and could be of instructional interest. Relevant instances arise from linear optimization formulations of combinatorial problems, such as set covering and set partitioning, which are degenerate and with small input data length.

In a 1980 technical report, reprinted as [9] with a postscript by Avis [1], Zadeh introduced and studied instances requiring an exponential number of iterations whose entries are small integers. In addition, Zadeh pointed out that his constructions, and many others requiring an exponential number of iterations, occur in so-called deformed products of polytopes. For more details about pivot based algorithms, instances requiring an exponential number of iterations for simplex methods, and related results, we refer to the surveys of Meunier [7], 
Terlaky and Zhang [8], and Ziegler [10], and to the recent results of Avis and Friedmann [2], and references therein.

\section{Two Small Degenerate Linear Optimization Instances}

Let $\mathbf{0}$ denote the origin, and $\mathbf{2}$ the vector whose coordinates are all equal to 2 . The linear optimization instance $\left(L O_{0}\right)$ considered by Kitahara and Mizuno in $[4,5]$, with $\boldsymbol{x} \in \boldsymbol{R}^{m}$, is:

$$
\begin{array}{ll}
\text { maximize } & \sum_{i=1}^{m} x_{i} \\
\text { subject to } & x_{1 \leq 1} \leq \text { for } k=2,3, \ldots, m \\
& 2 \sum_{i=1}^{k-1} x_{i}+x_{k} \leq 2^{k}-1 \quad \\
& \boldsymbol{x} \geq \mathbf{0}
\end{array}
$$

The feasible region of $\left(L O_{0}\right)$ is a Klee-Minty cube and the simplex method with Dantzig's pivoting rule visits all its vertices. Thus, $2^{m}-1$ iterations may be required to solve the standard form of $\left(L O_{0}\right)$ as observed by Kitahara and Mizuno [4, 5].

The first small linear optimization instance $\left(L O_{1}\right)$ is obtained from $\left(L O_{0}\right)$ by multiplying the first inequality of $\left(L O_{0}\right)$ by 2 , and setting to 2 the right-hand-side of the next $m-1$ inequalities:

$$
\begin{array}{ll}
\text { maximize } & \sum_{i=1}^{m} x_{i} \\
\text { subject to } & 2 x_{1} \leq 2 \\
& 2 \sum_{i=1}^{k-1} x_{i}+x_{k} \leq 2 \text { for } k=2,3, \ldots, m \\
& \boldsymbol{x} \geq \mathbf{0}
\end{array}
$$

One can check that the first $m-1$ inequalities of $\left(L O_{1}\right)$ are redundant, and that the feasible region of $\left(L O_{1}\right)$ is the simplex obtained by intersecting the positive orthant with the half-space defined by $2 \sum_{i=1}^{m-1} x_{i}+x_{m} \leq 2$. The vertices of this simplex are

$$
\left\{\boldsymbol{0}, \boldsymbol{e}_{1}, \boldsymbol{e}_{2}, \ldots, \boldsymbol{e}_{m-1}, 2 \boldsymbol{e}_{m}\right\}
$$

where $\boldsymbol{e}_{i}$ denotes the $i$-th unit vector of $\boldsymbol{R}^{m}$. Note that $\boldsymbol{e}_{1}$ is a highly degenerate vertex of degree $2 m-1$ as it satisfies with equality all the inequalities of $\left(L O_{1}\right)$ except $x_{1} \geq 0$. The standard form associated to $\left(L O_{1}\right)$, with slack variable $\boldsymbol{y} \in \boldsymbol{R}^{m}$, is:

$$
\begin{array}{ll}
\text { maximize } & \sum_{i=1}^{m} x_{i} \\
\text { subject to } & 2 x_{1}+y_{1}=2 \\
& 2 \sum_{i=1}^{k-1} x_{i}+x_{k}+y_{k}=2 \text { for } k=2,3, \ldots, m \\
& \boldsymbol{x} \geq \mathbf{0}, \boldsymbol{y} \geq \mathbf{0}
\end{array}
$$

The second small degenerate linear optimization instance $\left(\mathrm{LO}_{2}\right)$ is obtained from $\left(\mathrm{LO}_{0}\right)$ 
by setting to 0 the right-hand-side of the first $m$ inequalities:

$$
\begin{array}{ll}
\text { maximize } & \sum_{i=1}^{m} x_{i} \\
\text { subject to } & x_{1} \leq 0 \\
& 2 \sum_{i=1}^{k-1} x_{i}+x_{k} \leq 0 \quad \text { for } k=2,3, \ldots, m \\
& \boldsymbol{x} \geq \mathbf{0}
\end{array}
$$

One can check that the feasible region of $\left(\mathrm{LO}_{2}\right)$ is reduced to the origin $\mathbf{0}$ which forms the unique and highly degenerate optimal point. The standard form associated to $\left(\mathrm{LO}_{2}\right)$ is:

$$
\begin{array}{ll}
\text { maximize } & \sum_{i=1}^{m} x_{i} \\
\text { subject to } & x_{1}+y_{1}=0 \\
& 2 \sum_{i=1}^{k-1} x_{i}+x_{k}+y_{k}=0 \quad \text { for } k=2,3, \ldots, m \\
& \boldsymbol{x} \geq \mathbf{0}, \boldsymbol{y} \geq \mathbf{0}
\end{array}
$$

\section{Proposition 2.1.}

(i) For both $\left(\mathrm{LO}_{1}\right)$ and $\left(\mathrm{LO}_{2}\right)$, the entries of the constraint matrix, the right-hand-side vector, and the cost vector are $\{0,1,2\}$-valued.

(ii) The feasible region of $\left(L O_{1}\right)$ is a full dimensional simplex including a highly degenerate vertex, and that of $\left(\mathrm{LO}_{2}\right)$ is reduced to a highly degenerate point.

(iii) For $\left(L O_{1}^{*}\right)$, starting from $(\boldsymbol{x}, \boldsymbol{y})=(\mathbf{0}, \mathbf{2})$, the simplex method with Dantzig's pivoting rule visits exactly 3 distinct vertices, and makes $2^{m-1}+1$ iterations, including $2^{m-1}-1$ at a highly degenerate vertex.

(iv) For $\left(L O_{2}^{*}\right)$, starting from $(\boldsymbol{x}, \boldsymbol{y})=(\mathbf{0}, \mathbf{0})$, the simplex method with Dantzig's pivoting rule visits exactly 1 vertex, and makes $2^{m}-1$ iterations at this highly degenerate vertex.

\section{Proof of Proposition 2.1}

Items $(i)$ and $(i i)$ of Proposition 2.1 restate the features of $\left(L O_{1}\right)$ and $\left(L O_{2}\right)$. Item (iii) deals with the behaviour of the simplex method with Dantzig's pivoting rule for $\left(L O_{1}^{*}\right)$. We first outline the simplex pivot sequences for $\left(L O_{1}^{*}\right)$ with $m=3$; that is:

$$
\begin{array}{lrrr}
\operatorname{maximize} & x_{1}+x_{2}+x_{3} & \\
\text { subject to } & 2 x_{1} & +y_{1} & =2 \\
& 2 x_{1}+x_{2} & +y_{2} & =2 \\
& 2 x_{1}+2 x_{2}+x_{3} \quad+y_{3} & =2 \\
& x_{1}, x_{2}, x_{3}, y_{1}, y_{2}, y_{3} & \geq 0
\end{array}
$$

Setting $y_{1}, y_{2}$, and $y_{3}$ as initial basic variables, the first dictionary, or tableau, is:

$$
\begin{aligned}
& \begin{array}{rrrrr}
z & = & x_{1} & +x_{2} & +x_{3} \\
\hline y_{1} & = & 2 & -2 x_{1} &
\end{array} \\
& y_{2}=2-2 x_{1} \quad-x_{2} \\
& y_{3}=2 \quad-2 x_{1}-2 x_{2} \quad-x_{3}
\end{aligned}
$$


where nonnegativity conditions $\boldsymbol{x} \geq \mathbf{0}$ and $\boldsymbol{y} \geq \mathbf{0}$ are omitted, and $z$ represents the objective function. The reduced costs, i.e. the coefficients of nonbasic variables $x_{1}, x_{2}$, and $x_{3}$ in $z$, are positive. Thus, dual feasibility is not satisfied and the dictionary is not optimal.

The adopted pivoting rule is Dantzig's rule, and the minimum index rule is used in case of ties as follows:

The entering variable should be a nonbasic variable with the largest reduced cost. If two or more nonbasic variables have the largest reduced cost, the one with the smallest index is chosen.

The leaving variable should be a basic variable reaching 0 as the entering variable increases. If two or more basic variables reach 0 simultaneously, the one with the smallest index is chosen.

Applying this pivoting rule to the first dictionary, $x_{1}$ is the entering variable, $y_{1}$ is the leaving one, and the second dictionary is:

\begin{tabular}{rlrrrr}
$z$ & $=$ & 1 & $-\frac{y_{1}}{2}$ & $+x_{2}$ & $+x_{3}$ \\
\hline$x_{1}$ & $=$ & 1 & $-\frac{y_{1}}{2}$ & & \\
$y_{2}$ & $=$ & & $y_{1}$ & $-x_{2}$ & \\
$y_{3}$ & $=$ & & $y_{1}$ & $-2 x_{2}$ & $-x_{3}$
\end{tabular}

$x_{2}$ is the next entering variable, $y_{2}$ the leaving one, and the third dictionary is:

$$
\begin{aligned}
& \begin{array}{c}
z=1+\frac{y_{1}}{2}-y_{2}+x_{3} \\
\hline x_{1}=1-\frac{y_{1}}{2}
\end{array} \\
& x_{2}=y_{1}-y_{2} \\
& y_{3}=-y_{1}+2 y_{2}-x_{3}
\end{aligned}
$$

$x_{3}$ is the next entering variable, $y_{3}$ the leaving one, and the fourth dictionary is:

$$
\begin{aligned}
& \begin{array}{c}
z=1-\frac{y_{1}}{2} \quad+y_{2}-y_{3} \\
\hline x_{1}=1-\frac{y_{1}}{2}
\end{array} \\
& x_{2}=y_{1}-y_{2} \\
& x_{3}=-y_{1}+2 y_{2}-y_{3}
\end{aligned}
$$

$y_{2}$ is the next entering variable, $x_{2}$ the leaving one, and the fifth dictionary is:

$$
\begin{aligned}
& \begin{array}{c}
z=1+\frac{y_{1}}{2}-x_{2}-y_{3} \\
\hline x_{1}=1-\frac{y_{1}}{2}
\end{array} \\
& y_{2}=y_{1}-x_{2} \\
& x_{3}=y_{1}-2 x_{2}-y_{3}
\end{aligned}
$$

$y_{1}$ is the next entering variable, $x_{1}$ the leaving one, the sixth dictionary is:

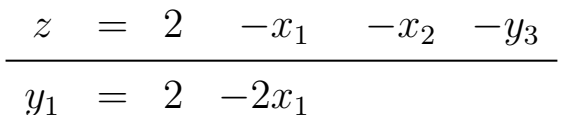

$$
\begin{aligned}
& y_{2}=2-2 x_{1} \quad-x_{2} \\
& x_{3}=2-2 x_{1}-2 x_{2}-y_{3}
\end{aligned}
$$


which is optimal as all reduced costs are nonpositive, and the optimal value is 2 .

The observed pivot sequence starts at the initial basic feasible solution $(\boldsymbol{x}, \boldsymbol{y})=(\mathbf{0}, \mathbf{2})$ with an objective value of 0 . The highly degenerate second basic feasible solution is $(\boldsymbol{x}, \boldsymbol{y})=$ $\left(\boldsymbol{e}_{1}, \mathbf{0}\right)$ with an objective value of 1 . The following $2^{2}-1$ basic feasible solutions remain at the same vertex with an objective value of 1 until the penultimate iteration. The last iteration reaches the optimal basic feasible solution $(\boldsymbol{x}, \boldsymbol{y})=\left(2 \boldsymbol{e}_{3}, \mathbf{2}-2 \boldsymbol{e}_{3}\right)$ with an objective value of 2 . This sequence of 5 simplex pivots is summarized in $\left(S_{1}^{3}\right)$ where 2 square blocks are highlighted to layout the recursive pattern followed by the sequence $\left(S_{1}^{m}\right)$ of the $2^{m-1}$ simplex pivots required for $m \geq 3$. The sequence $\left(S_{1}^{m}\right)$ is described in Proposition 3.1 which implies item (iii) of Proposition 2.1

\begin{tabular}{lllllll} 
iteration: & 0 & 1 & 2 & 3 & 4 & 5 \\
\hline \multirow{3}{*}{ basic variables: } & $y_{1}$ & $y_{2}$ & $x_{1}$ & $x_{1}$ & $x_{1}$ & $y_{1}$ \\
& $y_{2}$ & $x_{2}$ & $x_{2}$ & $y_{2}$ & $y_{2}$ \\
& $y_{3}$ & $y_{3}$ & $y_{3}$ & $x_{3}$ & $x_{3}$ & $x_{3}$
\end{tabular}

Proposition 3.1. For $m \geq 3$, the sequence $\left(S_{1}^{m}\right)$ of the $2^{m-1}+1$ pivots followed by the simplex method with Dantzig's pivoting rule for $\left(L O_{1}^{*}\right)$ satisfies:

(i) the basic variables at iteration 0 are $\left\{y_{1}, y_{2}, \ldots, y_{m}\right\}$; that is, the initial basic feasible solution is $(\boldsymbol{x}, \boldsymbol{y})=(\mathbf{0}, \mathbf{2})$,

(ii) the basic variables at iteration $2^{m-1}+1$ are $\left\{y_{1}, y_{2}, \ldots, y_{m-1}, x_{m}\right\}$,

(iii) $y_{m}$ remains a basic variable until iteration $2^{m-2}$ where it is replaced by $x_{m}$ which remains a basic variable until iteration $2^{m-1}+1$,

(iv) for iterations 1 to $2^{m-2}$, the basic variables are obtained by adding $y_{m}$ to the basic variables of $\left(S_{1}^{m-1}\right)$,

(v) for iterations $2^{m-2}+1$ to $2^{m-1}$, the basic variables are obtained by adding $x_{m}$ to the basic variables corresponding to the iterations $2^{m-2}$ to 1 of $\left(S_{1}^{m-1}\right)$.

Consequently, starting from the initial basic feasible solution $(\boldsymbol{x}, \boldsymbol{y})=(\mathbf{0}, \mathbf{2})$ with an objective value of 0 , the pivot sequence first reaches the highly degenerate second basic feasible solution $(\boldsymbol{x}, \boldsymbol{y})=\left(\boldsymbol{e}_{1}, \mathbf{0}\right)$ with an objective value of 1 . The following $2^{m-1}-1$ basic feasible solutions remain at the same vertex with an objective value of 1 until the penultimate iteration. The last iteration reaches the optimal basic feasible solution $(\boldsymbol{x}, \boldsymbol{y})=\left(2 \boldsymbol{e}_{m}, \mathbf{2}-2 \boldsymbol{e}_{m}\right)$ with an objective value of 2 . Thus, while visiting exactly 3 vertices, the simplex method with Dantzig's pivoting rule solves $\left(L O_{1}^{*}\right)$ by $2^{m-1}+1$ iterations - including $2^{m-1}-1$ iterations at a highly degenerate vertex.

Proof. The exponential number of iterations results from the fact that for any $k=2, \ldots, m$, $x_{k}$ becomes a basic variable for the first time at iteration $2^{k-2}+1$. This can be shown recursively by exploiting the lower triangular structure of the dictionaries. The analysis is essentially adapted from the one used by Kitahara and Mizuno [5] to show that $\left(\mathrm{LO}_{0}\right)$ requires $2^{m}-1$ iterations. Thus, we simply illustrate the recursive pattern from $\left(S_{1}^{m}\right)$ to $\left(S_{1}^{m+1}\right)$ for $m=2$ and 3 . One can check that $\left(S_{1}^{2}\right)$ is equal to:

\begin{tabular}{lcccc} 
iteration: & 0 & 1 & 2 & 3 \\
\hline \multirow{2}{*}{ basic variables: } & $y_{1}$ & $x_{1}$ & $x_{1}$ & $y_{1}$ \\
& $y_{2}$ & $y_{2}$ & $x_{2}$ & $x_{2}$
\end{tabular}


Note that the first highlighted block of $\left(S_{1}^{3}\right)$ corresponds to the iterations 1 and 2 of $\left(S_{1}^{2}\right)$, and that the second highlighted block of $\left(S_{1}^{3}\right)$ is the mirror image of the first highlighted block. Then, one can check that $\left(S_{1}^{4}\right)$ is equal to:

\begin{tabular}{lllllllllll} 
iteration: & 0 & 1 & 2 & 3 & 4 & 5 & 6 & 7 & 8 & 9 \\
\hline \multirow{4}{*}{ basic variables: } & $y_{1}$ & $y_{2}$ & $x_{1}$ & $x_{1}$ & $x_{1}$ & $x_{1}$ & $x_{1}$ & $x_{1}$ & $x_{1}$ & $y_{1}$ \\
& $y_{2}$ & $\boldsymbol{x}_{2}$ & $x_{2}$ & $y_{2}$ & $y_{2}$ & $x_{2}$ & $x_{2}$ & $y_{2}$ & $y_{2}$ \\
& $y_{3}$ & $y_{3}$ & $\boldsymbol{x}_{3}$ & $x_{3}$ & $x_{3}$ & $x_{3}$ & $y_{3}$ & $y_{3}$ & $y_{3}$ \\
& $y_{4}$ & $y_{4}$ & $y_{4}$ & $y_{4}$ & $y_{4}$ & $\boldsymbol{x}_{4}$ & $x_{4}$ & $x_{4}$ & $x_{4}$ & $x_{4}$
\end{tabular}

Note that the first highlighted block of $\left(S_{1}^{4}\right)$ corresponds to the iterations 1,2,3 and 4 of $\left(S_{1}^{3}\right)$, and that the second highlighted block of $\left(S_{1}^{4}\right)$ is the mirror image of the first highlighted block. The variable $x_{k}$ is bolded the first time it becomes basic; that is, at iteration $2^{k-2}+$ 1.

Item $(i v)$ of Proposition 2.1 deals with the behaviour of the simplex method with Dantzig's pivoting rule for $\left(L O_{2}^{*}\right)$. We first outline the simplex pivot sequences for $\left(L O_{2}^{*}\right)$ with $m=2$; that is:

$$
\begin{array}{lrl}
\operatorname{maximize} & x_{1}+x_{2} & \\
\text { subject to } & x_{1} & +y_{1}=0 \\
& 2 x_{1}+x_{2}+y_{2}=0 \\
& x_{1}, x_{2}, y_{1}, y_{2} \geq 0
\end{array}
$$

Setting $y_{1}$ and $y_{2}$ as initial basic variables, the first dictionary is:

$$
\begin{aligned}
& \begin{aligned}
z & =x_{1}+x_{2} \\
\hline y_{1} & =-x_{1}
\end{aligned} \\
& y_{2}=-2 x_{1}-x_{2}
\end{aligned}
$$

where nonnegativity conditions $\boldsymbol{x} \geq \mathbf{0}$ and $\boldsymbol{y} \geq \mathbf{0}$ are omitted, and $z$ represents the objective function. While $(\boldsymbol{x}, \boldsymbol{y})=(\mathbf{0}, \mathbf{0})$ corresponds to an optimal vertex, the reduced costs, i.e. the coefficients of nonbasic variables $x_{1}$ and $x_{2}$ in $z$, are positive. Thus, dual feasibility is not satisfied and the dictionary is not optimal. As for $\left(L O_{1}^{*}\right)$, the adopted pivoting rule is Dantzig's rule, and the minimum index rule is used in case of ties.

Applying the pivoting rule to the first dictionary, $x_{1}$ is the entering variable, $y_{1}$ is the leaving one, and the second dictionary is:

$$
\begin{array}{rll}
z & =-y_{1}+x_{2} \\
\hline x_{1} & =-y_{1} \\
y_{2} & =2 y_{1} & -x_{2}
\end{array}
$$

$x_{2}$ is the next entering variable, $y_{2}$ the leaving one, and the third dictionary is:

$$
\begin{array}{rll}
z & =y_{1}-y_{2} \\
\hline x_{1} & =-y_{1} \\
x_{2} & =2 y_{1}-y_{2}
\end{array}
$$

$y_{1}$ is the next entering variable, $x_{1}$ the leaving one, and the fourth dictionary is optimal as all the reduced costs are nonpositive, and the optimal value is 0 :

$$
\begin{array}{rll}
z & =-x_{1} & -y_{2} \\
\hline y_{1} & =-x_{1} & \\
x_{2} & =-2 x_{1} & -y_{2}
\end{array}
$$


The observed pivot sequence starts at the initial basic feasible solution $(\boldsymbol{x}, \boldsymbol{y})=(\mathbf{0}, \mathbf{0})$ with an objective value of 0 . The following $2^{2}-1$ basic feasible solutions remain at the same vertex with an objective value of 0 until reaching an optimal basis for the same solution $(\boldsymbol{x}, \boldsymbol{y})=(\mathbf{0}, \mathbf{0})$. Using an approach similar to the one used for item (iii) of Proposition 2.1, one can derive Proposition 3.2 which implies item $(i v)$ of Proposition 2.1.

Proposition 3.2. For $m \geq 3$, the sequence $\left(S_{2}^{m}\right)$ of the $2^{m}-1$ pivots followed by the simplex method with Dantzig's pivoting rule for $\left(\mathrm{LO}_{2}^{*}\right)$ satisfies:

(i) the basic variables at iteration 0 are $\left\{y_{1}, y_{2}, \ldots, y_{m}\right\}$; that is, the initial basic feasible solution is $(\boldsymbol{x}, \boldsymbol{y})=(\mathbf{0}, \mathbf{0})$,

(ii) the basic variables at iteration $2^{m}-1$ are $\left\{y_{1}, y_{2}, \ldots, y_{m-1}, x_{m}\right\}$,

(iii) $y_{m}$ remains a basic variable until iteration $2^{m-1}-1$ where it is replaced by $x_{m}$ which remains a basic variable until iteration $2^{m}-1$,

(iv) for iterations 0 to $2^{m-1}-1$, the basic variables are obtained by adding $y_{m}$ to the basic variables of $\left(S_{2}^{m-1}\right)$,

(v) for iterations $2^{m-1}$ to $2^{m}-1$, the basic variables are obtained by adding $x_{m}$ to the basic variables corresponding to the iterations $2^{m-1}-1$ to 0 of $\left(S_{2}^{m-1}\right)$.

Consequently, starting from the initial basic feasible solution $(\boldsymbol{x}, \boldsymbol{y})=(\mathbf{0}, \mathbf{0})$ with an objective value of 0 , the following $2^{m}-1$ basic feasible solutions remain at the same vertex with an objective value of 0 until reaching an optimal basis for the same solution $(\boldsymbol{x}, \boldsymbol{y})=(\mathbf{0}, \mathbf{0})$. Thus, while visiting exactly one vertex, the simplex method with Dantzig's pivoting rule solves $\left(\mathrm{LO}_{2}^{*}\right)$ by $2^{m}-1$ iterations at a highly degenerate vertex.

Proof. The exponential number of iterations results from the fact that for any $k=2, \ldots, m$, $x_{k}$ becomes a basic variable for the first time at iteration $2^{k-1}$. As for the proof of Proposition 3.1, we simply illustrate the recursive pattern from $\left(S_{2}^{m}\right)$ to $\left(S_{2}^{m+1}\right)$ for $m=2$. One can check that $\left(S_{2}^{2}\right)$ and $\left(S_{2}^{3}\right)$ are equal to:

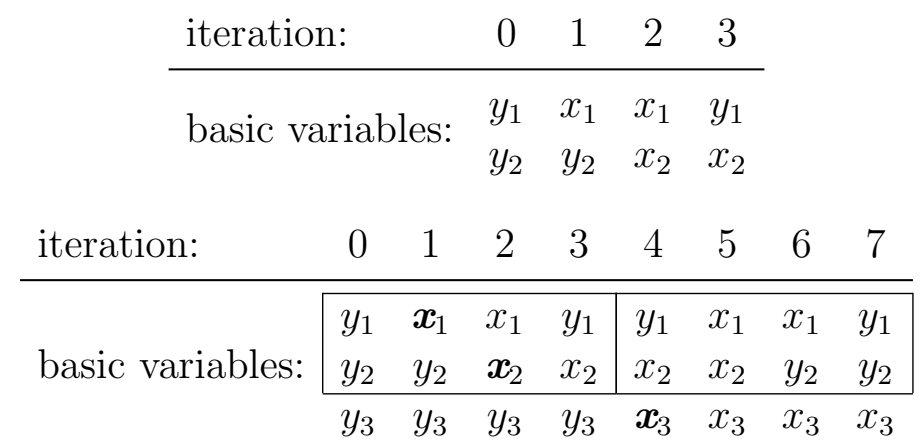

Note that the first highlighted block of $\left(S_{2}^{3}\right)$ corresponds to the iterations $0,1,2$, and 3 of $\left(S_{2}^{2}\right)$, and that the second highlighted block of $\left(S_{2}^{3}\right)$ is the mirror image of the first highlighted block. The variable $x_{k}$ is bolded the first time it becomes basic; that is, at iteration $2^{k-1}$.

\section{Acknowledgment}

The authors would like to thank the anonymous referees for valuable comments and suggestions. This research is supported in part by Grant-in-Aid for Science Research (A) 26242027, Young Scientists (Start-up) 15H06617 of Japan Society for the Promotion of Science, the 
Natural Sciences and Engineering Research Council of Canada Discovery Grant program (RGPIN-2015-06163), and by the Digiteo Chair C\&O program. This research was done in part while the authors were at the LRI, Université Paris-Sud, Orsay, within the Digiteo invited researchers program, and in part while the third author visited the Tokyo Institute of Technology.

\section{References}

[1] D. Avis: Postscript to "What is the worst case behavior of the simplex algorithm?" In D. Avis, D. Bremner, and A. Deza (eds.): Polyhedral Computation (Centre de Recherches Mathématiques Series, Montréal, 2009), 145-147.

[2] D. Avis and O. Friedmann; D. Avis and O. Friedmann: An exponential lower bound for Cunningham's rule. Mathematical Programming, 161 (2017), 271-305.

[3] G.B. Dantzig: Linear Programming and Extensions (Princeton University Press, Princeton, 1963).

[4] T. Kitahara and S. Mizuno: Klee-Minty's LP and upper bounds for Dantzig's simplex method. Operations Research Letters, 39 (2011), 88-91.

[5] T. Kitahara and S. Mizuno: Lower bounds for the maximum number of solutions generated by the simplex method. Journal of the Operations Research Society of Japan, 54 (2011), 191-200.

[6] V. Klee and G.J. Minty: How good is the simplex algorithm? In O. Shisha (ed.): Inequalities III (Academic Press, New York, 1972), 159-175.

[7] F. Meunier: Computing and proving with pivots. RAIRO Operations Research, 47 (2013), 331-360.

[8] T. Terlaky and S. Zhang: Pivot rules for linear programming: A survey on recent theoretical developments. Annals of Operations Research, 46 (1993), 203-233.

[9] N. Zadeh: What is the worst case behavior of the simplex algorithm? In D. Avis, D. Bremner, and A. Deza (eds.): Polyhedral Computation (Centre de Recherches Mathématiques Series, Montréal, 2009), 131-143.

[10] G.M. Ziegler: Typical and extremal linear programs. In M. Grötschel (ed.): The Sharpest Cut: The Impact of Manfred Padberg and His Work (MPS-SIAM Series on Optimization, Philadelphia, 2004), 217-230.

\section{A. Appendix}

This section complements the sketch of the proofs for Propositions 3.1 and 3.2. Since the proofs are very similar, we only detail the proof of Proposition 3.2.

The sequence $\left(S_{2}^{m}\right)$ of basic feasible solutions generated by the simplex method with Dantzig's pivoting rule for the problem $\left(\mathrm{LO}_{2}^{*}\right)$ satisfies the following properties:

(i) the basic variables at iteration 0 are $\left\{y_{1}, y_{2}, \ldots, y_{m}\right\}$; that is, the initial basic feasible solution is $(\boldsymbol{x}, \boldsymbol{y})=(\mathbf{0}, \mathbf{0})$,

(ii) the number of iterations is $2^{m}-1$,

(iii) the reduced cost of any dictionary is $\{-1,1\}$-valued,

(iv) the basic variables at iteration $2^{m}-1$ are $\left\{y_{1}, \ldots, y_{m-1}, x_{m}\right\}$; that is, the optimal basic feasible solution is $(\boldsymbol{x}, \boldsymbol{y})=(\mathbf{0}, \mathbf{0})$. 
Proof. The properties are shown inductively. As noted in the sketch of the proof of Proposition 3.2, the properties hold for $m=2$. Let assume the properties hold until $k$ and consider the sequence $\left(S_{2}^{k+1}\right)$. The initial dictionary, associated to the initial solution $(\boldsymbol{x}, \boldsymbol{y})=(\mathbf{0}, \mathbf{0})$ with basic variables $\left\{y_{1}, y_{2}, \ldots, y_{k+1}\right\}$, is:

$$
\begin{array}{rrrrrr}
z & = & x_{1} & \cdots & +x_{k} & +x_{k+1} \\
y_{1} & = & -x_{1} & & & \\
& \vdots & & & & \\
y_{k} & = & -2 x_{1} & \cdots & -x_{k} & \\
y_{k+1} & = & -2 x_{1} & \cdots & -2 x_{k} & -x_{k+1}
\end{array}
$$

Note that the nonbasic variable $x_{k+1}$ appears only in the objective function and in the last constraint of $\left(D_{0}\right)$. As its reduced cost is $1, x_{k+1}$ is not chosen as an entering variable by Dantzig's pivoting rule until all other reduced costs are less than 1 . Furthermore, $y_{k+1}$ is not chosen as a leaving variable when other basic variable can be chosen, since each right hand side constant is zero and the minimum index rule is used in case of ties. While the variable $x_{k+1}$ remain nonbasic, the dictionaries are essentially, i.e. except for the last row and column, in a one-to-one correspondence with the dictionaries associated to $\left(S_{2}^{k}\right)$. Note that the reduced costs are $\{-1,1\}$-valued and that they, except the one for $x_{k+1}$, become less than 1 precisely when a dictionary associated to an optimal basic feasible solution of $\left(S_{2}^{k}\right)$ is obtained. In other words, the first $2^{k}-1$ iterations of $\left(S_{2}^{k+1}\right)$ can be associated to the $2^{k}-1$ iterations of $\left(S_{2}^{k}\right)$. The next dictionary; that is, the dictionary at iteration $2^{k}$ with basic variables $\left\{y_{1}, \ldots, y_{k-1}, x_{k}, y_{k+1}\right\}$, is equal to:

$$
\begin{aligned}
& z=-x_{1} \quad \cdots \quad-y_{k}+x_{k+1} \\
& y_{1}=-x_{1} \\
& \vdots \\
& x_{k}=-2 x_{1} \cdots \quad-y_{k} \\
& y_{k+1}=2 x_{1} \cdots+2 y_{k}-x_{k+1}
\end{aligned}
$$

At this iteration, $x_{k+1}$ is chosen as the entering variable, and the next dictionary is equal to:

$$
\begin{array}{rrrrrr}
z & = & x_{1} & \cdots & +y_{k} & -y_{k+1} \\
y_{1} & = & -x_{1} & & & \\
& \vdots & & & & \\
x_{k} & = & -2 x_{1} & \cdots & -y_{k} & \\
x_{k+1} & = & 2 x_{1} & \cdots & +2 y_{k} & -y_{k+1}
\end{array}
$$

Note that reduced cost is $\{-1,1\}$-valued. The nonbasic variable $y_{k+1}$ appears only in the objective function and in the last constraint of $\left(D_{2^{k}+1}\right)$. As its reduced cost is $-1, y_{k+1}$ is not chosen as an entering variable by Dantzig's pivoting rule until the last pivot. Furthermore, $x_{k+1}$ is not chosen as a leaving variable when other basic variable can be chosen, since each right hand side constant is zero and the minimum index rule is used in case of ties. Hence, the last $2^{k}-1$ iterations of $\left(S_{2}^{k+1}\right)$ can be associated to the $2^{k}-1$ iterations of $\left(S_{2}^{k}\right)$. Note that the dictionary $\left(D_{2^{k}+1}\right)$ is, except for the last row and column, the same as dictionary $\left(D_{0}\right)$ where $y_{k}$ and $x_{k}$ are swapped. Thus, the optimal dictionary; that is, the dictionary at 
iteration $2^{k+1}$ with basic variables $\left\{y_{1}, \ldots, y_{k}, x_{k+1}\right\}$, is equal to:

$$
\begin{array}{rcccccc}
z & = & -x_{1} & \cdots & -x_{k} & -y_{k+1} & \\
y_{1} & = & -x_{1} & & & & \\
& \vdots & & & & & \left(D_{2^{k+1}-1}\right) \\
y_{k} & = & -2 x_{1} & \cdots & -x_{k} & & \\
x_{k+1} & = & -2 x_{1} & \cdots & -2 x_{m} & -y_{k+1} &
\end{array}
$$

Hence, the properties hold for $k+1$.

Noriyoshi Sukegawa

Department of Information and System Engineering Faculty of Science and Engineering, Chuo University 3602, 1-13-27 Kasuga

Bunkyo-ku, Tokyo 112-8551, JAPAN

E-mail: sukegawa@ise.chuo-u.ac.jp 